\title{
Technical Note \\ Fully developed combined convection in a seven-rod horizontal bundle
}

\author{
S.C. Haldar \\ Department of Mechanical Engineering, Regional Engineering College, Rourkela-769 008, Orissa, India
}

\begin{abstract}
Numerical studies of combined free and forced convection through a seven-rod bundle in a circular shell in the horizontal configuration are reported in the paper. Each of the six peripheral circular rods has been modelled by an equivalent curved trapezium. The model has been verified to corroborate experimentally measured transport rate values. Buoyancy induces secondary motions in the transverse direction over the forced axial flow. The secondary velocities reduce the through-flow rate at a given impressed pressure gradient. The friction factor is consequently increased. The change in $f R e$ is perceptible for $G r$ defined on the rod radius more than approximately $10^{3}$, whereas $N u$ increases monotonically with $G r$.
\end{abstract}

\section{Introduction}

The study of mixed convection in a horizontal bundle is beset with complexities due to the fact that the buoyancy acts in a direction transverse to the throughflow. Even for a fully developed situation, buoyancy introduces non-zero transverse velocity components, ordinarily known as secondary velocities and the flow field becomes three-dimensional.

The interacting thermal-hydraulic fields become extremely complex when more than one heated body are present inside a shell. The buoyant flow from the lowermost surface sweeps over the upper ones which are also sources of additional transverse velocities. The objective of the present study is to analyse the flow and temperature fields in a bundle of seven heated rods contained in an adiabatic circular shell. The geometry replicates small heat exchangers and can be

E-mail address: imagined to simulate a nuclear reactor of CANDU design.

Although very little is reported in the literature on the geometry of the present interest, the information available on concentric annuli are of relevance.

Kaviany [1] reported a numerical solution of laminar fully developed combined convection in a horizontal concentric annulus of radius ratio 1.25 with a constant heat flux inner wall and an adiabatic outer shell. The results showed changes in lateral flow structures from a single to multiple cells as the Rayleigh number was increased. Nieckele and Patankar [2] carried out a similar study but for a wide range of radius ratios varying from 1.5 to 5.0. The study of mixed convection in horizontal annuli was preceded by that in plain tubes and most of these studies have been cited in [2].

Warrington and Crupper [3] studied experimentally the free convection in a fixed array of four isothermal cylinders placed in an isothermally cooled cubical enclosure for both horizontal and vertical orientations. It was observed that the vertical configuration convected less heat than the horizontal one. A numerical 


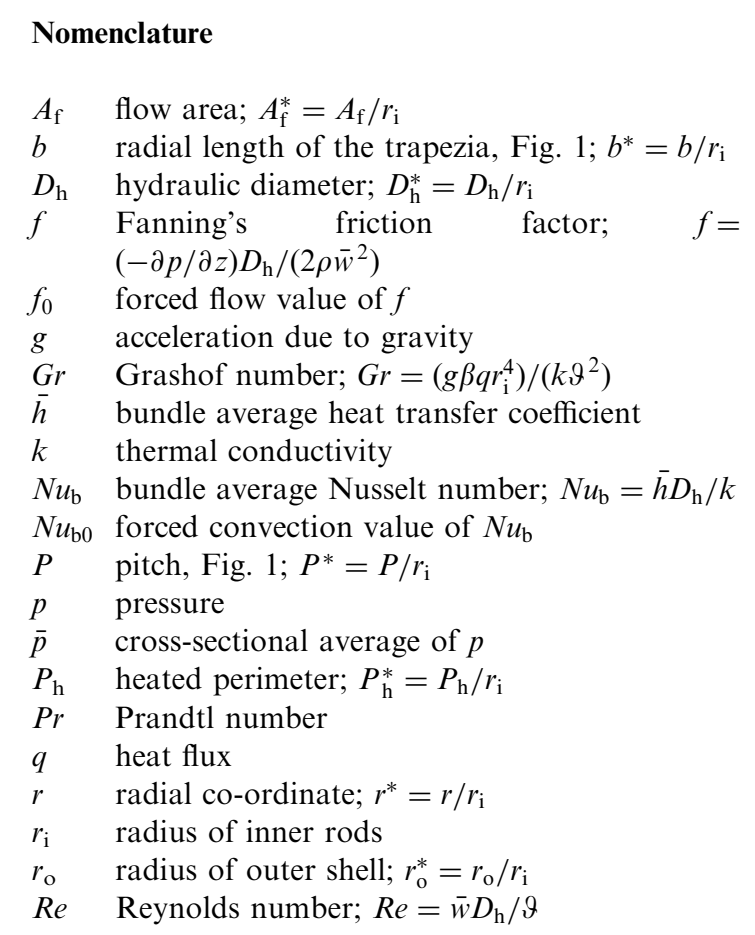

$R e^{\prime} \quad$ Reynolds number; $R e^{\prime}=\bar{w} r_{\mathrm{i}} / \vartheta$

$T \quad$ temperature; $T^{*}=\left(T-T_{\mathrm{b}}\right) / T_{\text {ref }}$

$T_{\mathrm{b}} \quad$ fluid bulk temperature

$\bar{T}_{\mathrm{r}} \quad$ average surface temperature of seven rods

$T_{\text {ref }} \quad$ reference temperature; $T_{\text {ref }}=q r_{\mathrm{i}} / k$

$u \quad$ radial velocity; $u^{*}=u /\left(\vartheta / r_{\mathrm{i}}\right)$

$v \quad$ angular velocity; $v^{*}=v /\left(\vartheta / r_{\mathrm{i}}\right)$

$w \quad$ axial velocity; $w^{*}=w /\left[r_{\mathrm{i}}^{2}(-\mathrm{d} \bar{p} / \mathrm{d} z) / \mu\right]$

$\bar{w} \quad$ cross-sectional average value of $w$

$z \quad$ axial co-ordinate; $z^{*}=z /\left(\operatorname{Re}^{\prime} r_{\mathrm{i}}\right)$

Greek symbols

$\beta \quad$ volumetric co-efficient of thermal expansion

$\mu \quad$ dynamic viscosity

$\vartheta \quad$ kinematic viscosity

$\phi \quad$ subtended angle of the trapezia, Fig. 1

$\psi \quad$ stream function; $\psi^{*}=\psi / \vartheta$

$\Omega \quad$ vorticity about the $z$-axis; $\Omega^{*}=\Omega /\left(\vartheta / r_{i}^{2}\right)$

$\theta \quad$ angular co-ordinate

\section{Superscript}

* dimensionless quantity study of free convection of air around two horizontal, differentially heated cylinders confined to an adiabatic circular enclosure was reported by Ho et al. [4]. They concluded a strong dependency of the fluid flow structure and heat transfer characteristics on the Rayleigh number, the inclination angle and the gap between the cylinders. It is believed that the present study of combined convection will lead to further information on the interacting velocity and temperature fields in a complex geometry.

\section{Physical model}

The seven-rod bundle in a circular shell is depicted in Fig. 1. The co-ordinate system and the corresponding velocity components are marked in the figure. The rods are under uniform heat flux condition, whereas the shell is adiabatic. The $r, \theta$ grid lines will intersect the surfaces of each of the six peripheral rods non-orthogonally. In order to avoid the associated complexities, the circular geometry of each of the six peripheral rods has been replaced by equivalent curved trapezoid maintaining the hydraulic diameter and the spacings between the rods (pitch) of the actual bundle. The size of the trapezoid was chosen by imposing an additional condition that the sums of the opposite sides of the trapezoid are equal. These yielded

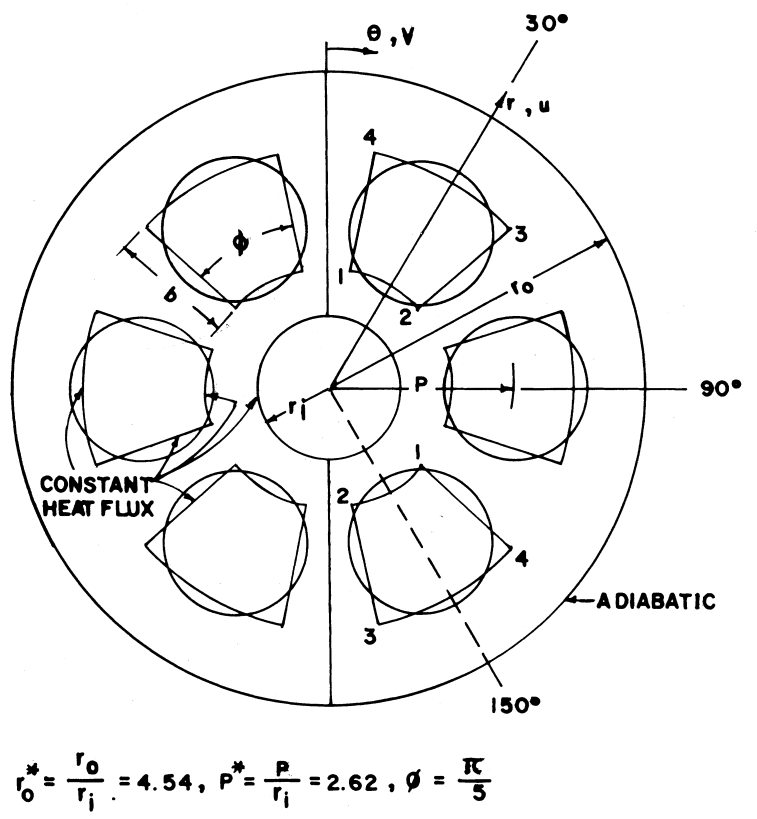

Fig. 1. Physical model of seven rods in a circular shell. 
$6 b^{* 2}+12 A_{1} b^{*}-\left(A_{2}-A_{1} A_{3}\right)=0$

and

$\phi=b^{*} / P^{*}$

where $A_{1}=\left(r_{\mathrm{o}}^{* 2}-7\right) /\left(r_{\mathrm{o}}^{*}+7\right), \quad A_{2}=\pi\left(r_{\mathrm{o}}^{* 2}-1\right), \quad A_{3}=$ $\pi\left(r_{\mathrm{o}}^{*}+1\right)$.

The solution of Eqs. (1) and (2) for $b^{*}$ and $\phi$ determines the size of the trapezoid, Fig. 1.

The heated perimeter and the through-flow area are modified to

$P_{\mathrm{h}}^{*}=2 \pi+24 b^{*}$

$A_{\mathrm{f}}^{*}=\pi\left(r_{\mathrm{o}}^{* 2}-1\right)-6 b^{* 2}$

Forced convection calculations carried out on the basis of the present model for $r_{\mathrm{o}}^{*}=4.0, P^{*}=2.70$ and for $r_{\mathrm{o}}^{*}=5.5, P^{*}=3.64$; when compared with literature information [5,6]; indicated agreement of the $f R e$ within $1 \%$ and $N u$ within $7 \%$.

An alternate model of equal flow area and minimum deviation in the heated perimeter also yielded agreement within similar limits.

The equal hydraulic diameter modelling was adopted for the flow and temperature field computation of the present geometry of $r_{\mathrm{o}}^{*}=4.54$ and $P^{*}=2.62$. The heated perimeter of the model becomes $4.11 \%$ higher than that of the true geometry.

\section{Analysis}

The flow and temperature fields are considered axially fully developed. However, transverse velocity components exist due to buoyancy effects. The transverse pressure gradients from $r$ and $\theta$ momentum equations were eliminated by cross differentiations to yield the transport equation on $\Omega$, the vorticity about the $z$-axis, where

$\Omega=\frac{\partial v}{\partial r}+\frac{v}{r}-\frac{1}{r} \frac{\partial u}{\partial \theta}$

Eq. (4) when expressed in terms of stream-function yields

$\nabla^{2} \psi=-\Omega \quad$ where the operator

$$
\nabla^{2}=\frac{\partial^{2}}{\partial r^{2}}+\frac{1}{r} \frac{\partial}{\partial r}+\frac{1}{r^{2}} \frac{\partial^{2}}{\partial \theta^{2}}
$$

All the four dimensionless governing equations are elliptic and have the general form

$C \frac{\mathrm{D} f}{\mathrm{D} t}=\nabla^{2} f+F \quad$ where $\frac{\mathrm{D}}{\mathrm{D} t}=u^{*} \frac{\partial}{\partial r^{*}}+\frac{v^{*}}{r^{*}} \frac{\partial}{\partial \theta}$
$C$ and $F$ for the different equations are tabulated below.

\begin{tabular}{llll}
\hline Equation & $f$ & $C$ & $F$ \\
$\begin{array}{l}\text { Vorticity- } \\
\text { transport }\end{array}$ & $\Omega^{*}$ & 1 & $G r\left[\frac{\cos \theta}{r^{*}} \frac{\partial T^{*}}{\partial \theta}+\sin \theta \frac{\partial T^{*}}{\partial r^{*}}\right]$ \\
$\begin{array}{l}\text { Stream- } \\
\text { function }\end{array}$ & $\psi^{*}$ & 0 & $\Omega *$ \\
$z$-Momentum & $w^{*}$ & 1 & 1 \\
Energy & $T^{*}$ & $\operatorname{Pr}$ & $-\frac{w^{*}}{\bar{w}^{*}} \frac{P_{\mathrm{h}}^{*}}{A_{\mathrm{f}}^{*}}$
\end{tabular}

The average axial velocity is given by

$\bar{w}^{*}=\frac{1}{A_{\mathrm{f}}^{*}} \int_{A_{\mathrm{f}}^{*}} w^{*} \mathrm{~d} A_{\mathrm{f}}^{*}$

While arriving at Eq. (7), it was assumed that $\partial p / \partial z$ in the $z$-momentum equation equals $\mathrm{d} \bar{p} / \mathrm{d} z$, where $\bar{p}$ is the cross-sectional average of $p$, and this has been shown to be valid for $G r / R e^{2}<1$ [2]. Thermally developed flow with uniform heat flux condition yielded $\partial T^{*} / \partial z^{*}$ $=\mathrm{d} T_{\mathrm{b}}^{*} / \mathrm{d} z^{*}=P_{\mathrm{h}}^{*} /\left(\operatorname{Pr} \cdot A_{\mathrm{f}}^{*}\right)$ in the energy equation.

\section{Boundary conditions}

The coupled governing equations were solved satisfying the following conditions on the solid boundaries and the symmetry lines.

Central rod: $u^{*}=v^{*}=w^{*}=\psi^{*}=0, \Omega^{*}=-\frac{\partial^{2} \psi^{*}}{\partial r^{* 2}}$ and $\frac{\partial T^{*}}{\partial r^{*}}=-1 \quad$ (uniform heat flux)

Outer shell: $u^{*}=v^{*}=w^{*}=\psi^{*}=0, \Omega^{*}=-\frac{\partial^{2} \psi^{*}}{\partial r^{* 2}}$ and

$$
\frac{\partial T^{*}}{\partial r^{*}}=0(\text { adiabatic })
$$

Symmetry lines $(\theta=0$ and $\pi)$ :

$$
\frac{\partial \psi^{*}}{\partial \theta}=\frac{\partial w^{*}}{\partial \theta}=\frac{\partial T^{*}}{\partial \theta}=\psi^{*}=\Omega^{*}=0
$$




\section{Peripheral rods}

Surface 12: $u^{*}=v^{*}=w^{*}=\psi^{*}=0, \Omega^{*}=-\frac{\partial^{2} \psi^{*}}{\partial r^{* 2}}$ and $\frac{\partial T^{*}}{\partial r^{*}}=+1$

Surface 43: $u^{*}=v^{*}=w^{*}=\psi^{*}=0, \Omega^{*}=-\frac{\partial^{2} \psi^{*}}{\partial r^{* 2}}$ and $\frac{\partial T^{*}}{\partial r^{*}}=-1$

Surface 14: $u^{*}=v^{*}=w^{*}=\psi^{*}=0, \Omega^{*}=-\frac{1}{r^{* 2}} \frac{\partial^{2} \psi^{*}}{\partial \theta^{2}}$ and $\frac{\partial T^{*}}{\partial \theta}=+1$

Surface 23: $u^{*}=v^{*}=w^{*}=\psi^{*}=0, \Omega^{*}=-\frac{1}{r^{* 2}} \frac{\partial^{2} \psi^{*}}{\partial \theta^{2}}$ and $\frac{\partial T^{*}}{\partial \theta}=-1$

\section{Solution}

The finite difference forms of each of the governing equations were solved by Successive Over Relaxation (SOR) technique satisfying the aforementioned boundary conditions. The salient features are enumerated below.

1. Convective terms in Eqs. (5) and (8) were expressed using upwinding scheme.

2. A grid independence test was carried out for pure forced convection condition with uniform grid sizes of $46 \times 30,46 \times 60$ and $92 \times 60$ in the $r$ and $\theta$ directions. The corresponding bundle average fRe and $\mathrm{Nu}$ values were 21.140, 24.534, 24.501 and 1.644, $1.529,1.539$, respectively. A grid size of $46 \times 60$ was chosen for the mixed convection solution to optimise between the accuracy and computation time.

3. Relaxation factors were varied from 0.6 to 1.8 for Gr decreasing from $10^{6}$ to 0 .

4. Average axial velocity, Eq. (9), was obtained by trapezoidal averaging of the nodal values weighted by the respective areas at every iteration cycle.

5. Temperatures on the solid surfaces were evaluated by one-sided second-order finite difference expressions of the respective thermal boundary conditions.

6. The vorticity on the solid surfaces reduces to $\Omega_{w}^{*}=-2 \psi_{n}^{*} / \Delta h^{* 2}$, where $\psi_{n}^{*}$ is the value of $\psi^{*}$ at the neighbouring node and $\Delta h^{*}$ is the corresponding grid size.

7. The individual equations were converged to $0.01 \%$. The solution was progressed till the wall vorticity and the wall temperatures were converged to $0.001 \%$ between two consecutive cycles of iteration where one cycle consists of solution of Eq. (5) for $\Omega^{*}$; Eq. (6) for $\psi^{*}$ and $u^{*}, v^{*}$ from $\psi^{*}$; Eq. (7) for $w^{*}$; and Eq. (8) for $T^{*}$.

\section{Experiments}

Numerical results have been generated for the details of the flow and temperature fields in addition to the transport rate values. The appropriateness of the geometrical modelling was sought by verifying salient information against experiments in a seven-rod horizontal bundle.

The experimental set-up consisted of three identical test sections, each comprising of seven rods, $12 \mathrm{~mm}$ OD and $1200 \mathrm{~mm}$ long, contained in a $54.5 \mathrm{~mm}$ ID and $4 \mathrm{~mm}$ thick plexiglass circular shell. The rod and shell arrangement offered a $r_{\mathrm{o}}^{*}$ of 4.54 and a $P^{*}$ of 2.62. The details of the set-up, instrumentation and transport phenomena in the vertical legs have been reported in Mohanty et al. [7]. The same set-up was used to measure the average transport rate values in the horizontal bundle. Each of the rods was, in fact, a stainless steel tube with imbedded heating elements and thermocouples. Separate, but equal, electric power was fed to each rod simulating uniform heat flux conditions.

Experiments were carried out at three $R e$ values of 565,856 and 1275; while the $G r$, based on the rod radius, was varied from $1.7 \times 10^{2}$ to $2.2 \times 10^{3}$. The heat transfer rate measured at a given $R e$ was normalised with respect to the corresponding forced convection value, $N u_{\mathrm{b}} / N u_{\mathrm{b} 0}$. Numerical results for the experimental geometry are compared with the measured values in Fig. 2. The good agreement observed vindicates that the geometric modelling through substituted trapezia is reproducing the gross transport rate values. Details of the flow and temperature fields were then studied numerically and the salient results are discussed below.

\section{Results and discussions}

The dimensionless conservation equations are independent of $R e$ and the numerical results were generated for $G r$ varying from 0 to $10^{6}$ and $P r=0.71$. The 


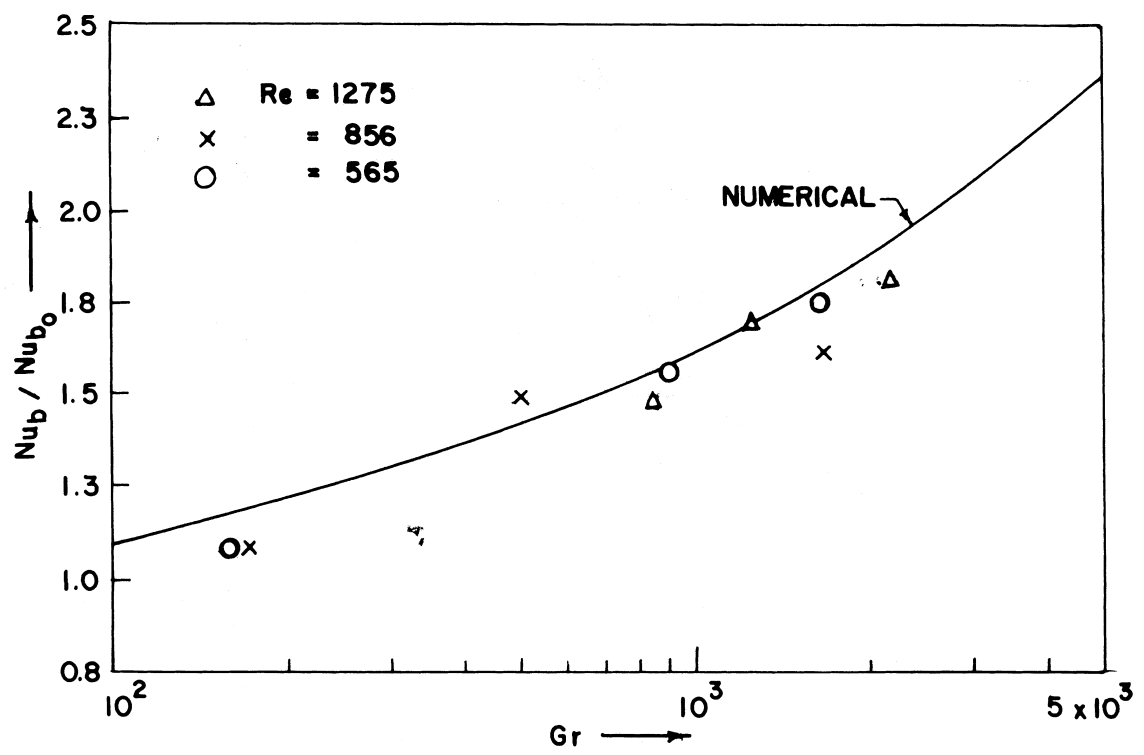

Fig. 2. Buoyancy effects on bundle average Nusselt number, $\operatorname{Pr}=0.71$; comparison with theory.

pure forced convection values are obtained as $f R e_{0}=$ 24.534 and $N u_{\mathrm{b} 0}=1.529$ for $G r=0$.

\subsection{Velocity components}

Buoyancy induces the radial and angular velocity components, $u^{*}$ and $v^{*}$, over the forced axial component, $w^{*}$.

The variations of $v^{*}$ with $r^{*}$ for different angular locations i.e. $\theta=30,90$ and $150^{\circ}$ have been presented in Fig. 3 for $G r=10^{6}$. The zero velocity line in the middle is due to the presence of the peripheral rods. The profiles in the gap between the central and the peripheral rods are spatially periodic. The fluid near

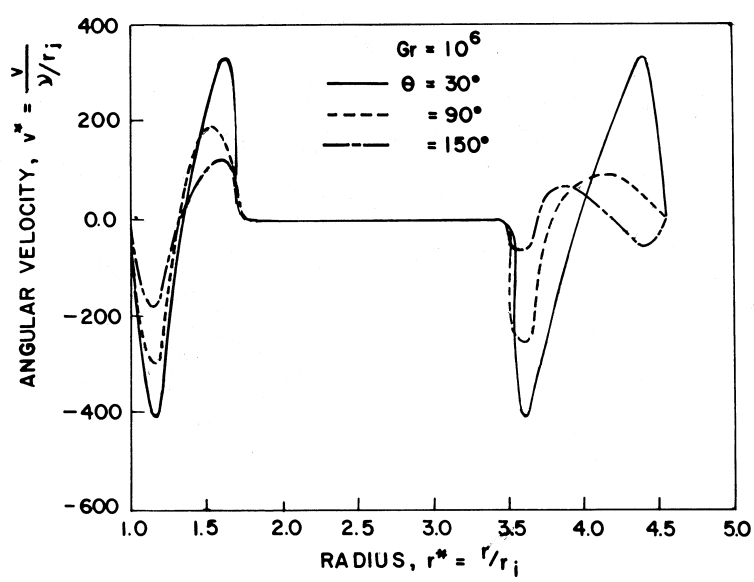

Fig. 3. Angular velocity profiles at various angles. the hot central rod rises along it, identified by decreasing $\theta$, causing the negative loop. The buoyant flow returns along the inner side (surface 12, Fig. 1) of the peripheral rods resulting in the positive loop.

The angular velocity variations between the peripheral rods and the outer shell are explained in the same manner. The fluid here rises along the outer side (surface 34 ) of the heated peripheral rods and comes down along the adiabatic outer shell. Note that the profile in the second gap for $\theta=150^{\circ}$ has two negative loops separated by a relatively large positive loop. This is due to the fact that the fluid there rises along the outer side of the peripheral rod as well as the shell, resulting

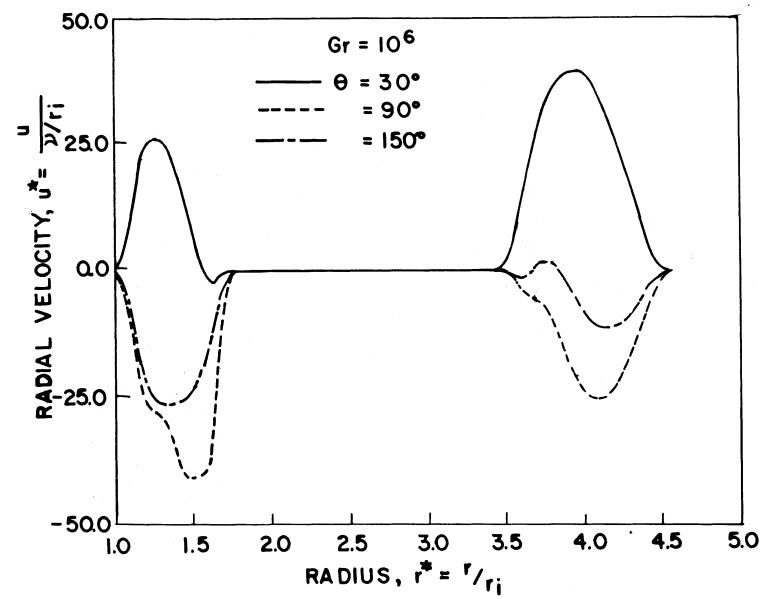

Fig. 4. Radial velocity profiles at various angles. 
in the two negative loops and comes down through the middle of the gap and, hence, the positive loop.

The profiles for the radial component of velocity, $u^{*}$ are plotted in Fig. 4 for the identical $G r$ and $\theta$ values of Fig. 3. Unlike $v^{*}$, the radial component is distinguished by single velocity cups. Furthermore, the variation for $\theta=30^{\circ}$ is all positive and opposite to those for $\theta=90$ and $150^{\circ}$. Fluid from the lower zones, e.g. $\theta=90$ and $150^{\circ}$, move inward from the outer shell to the heated rods, identified by decreasing radius and, therefore, negative values; whereas in the upper portion of the bundle, e.g. $\theta=30^{\circ}$, the fluid moves away from the rod resulting in the positive values. We also note by comparison with Fig. 3 that the radial velocity is about an order of magnitude lower than the angular velocity.

The effects of buoyancy on the axial velocity profiles have been shown in Figs. 5(a) and (b) for two angular locations of $\theta=0$ and $180^{\circ}$ i.e. upper and lower vertical symmetry lines. With increasing $G r$, the peaks are

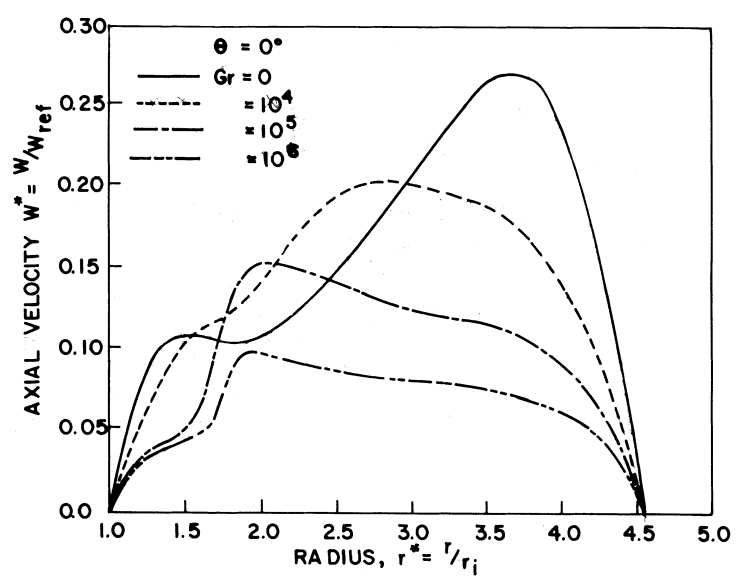

(a)

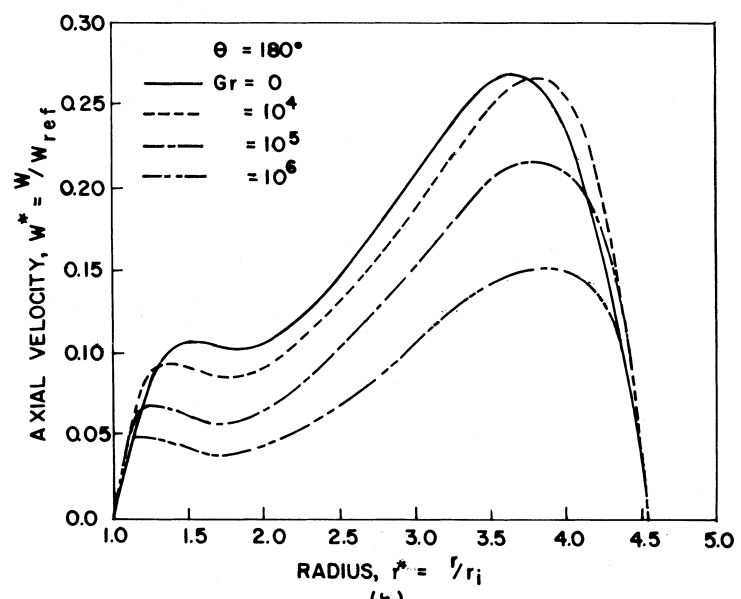

(b)

Fig. 5. Buoyancy effects on axial velocity profiles. observed to decrease, thereby reducing the throughflow rate for the same impressed axial pressure gradient. The profiles at $\theta=0^{\circ}$ shift toward the central rod with increase in $G r$, whereas those at $\theta=180^{\circ}$ shift toward the shell. This may be explained by the fact that the secondary fluid adjacent to the $\theta=0^{\circ}$ line moves towards the central rod, Fig. 6, thus pushing the axial component; while the direction of secondary flow adjacent to $\theta=180^{\circ}$ line is outwards and, hence, the corresponding shifting.
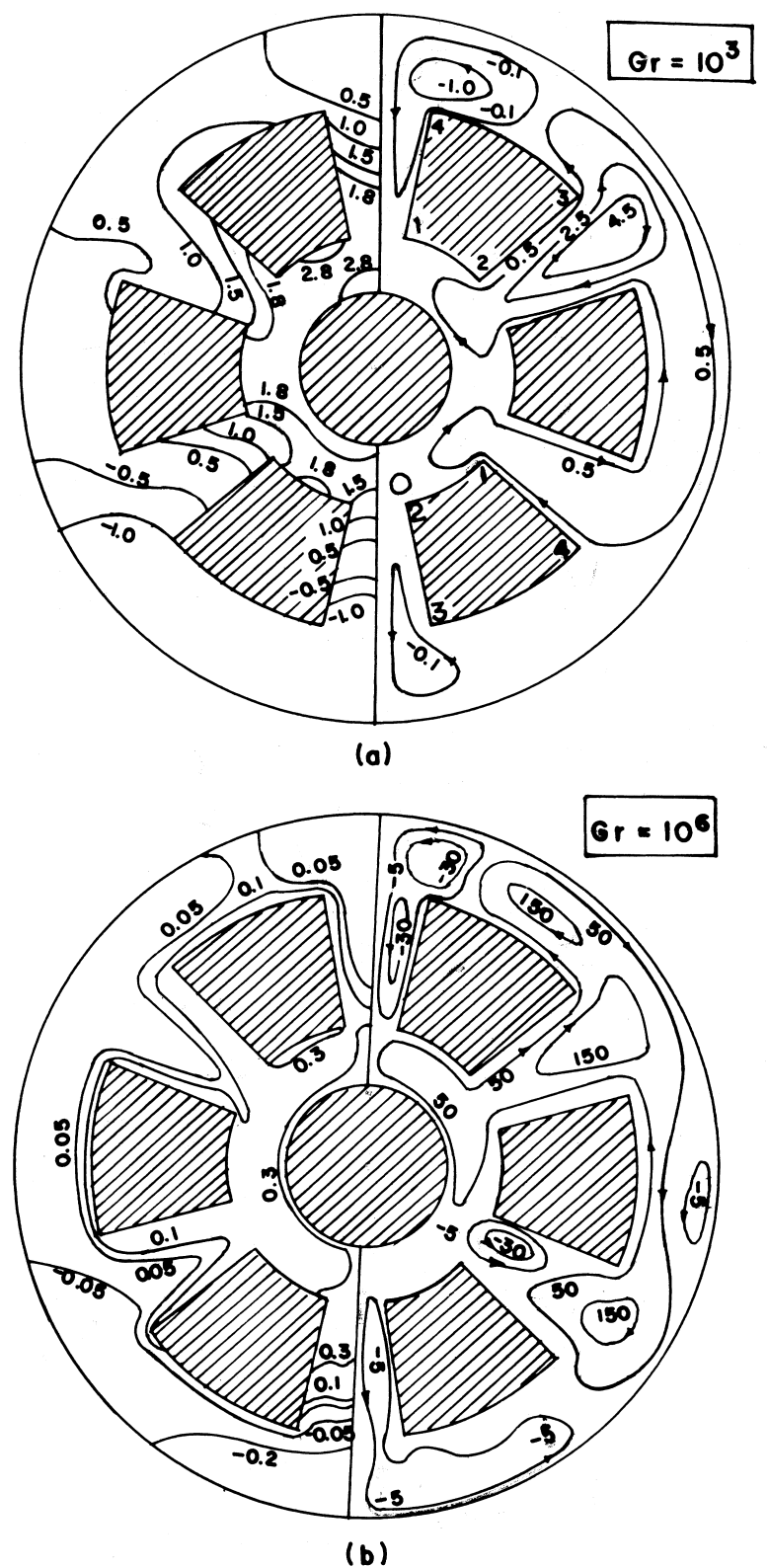

Fig. 6. Isotherms and streamlines in a seven-rod bundle. 


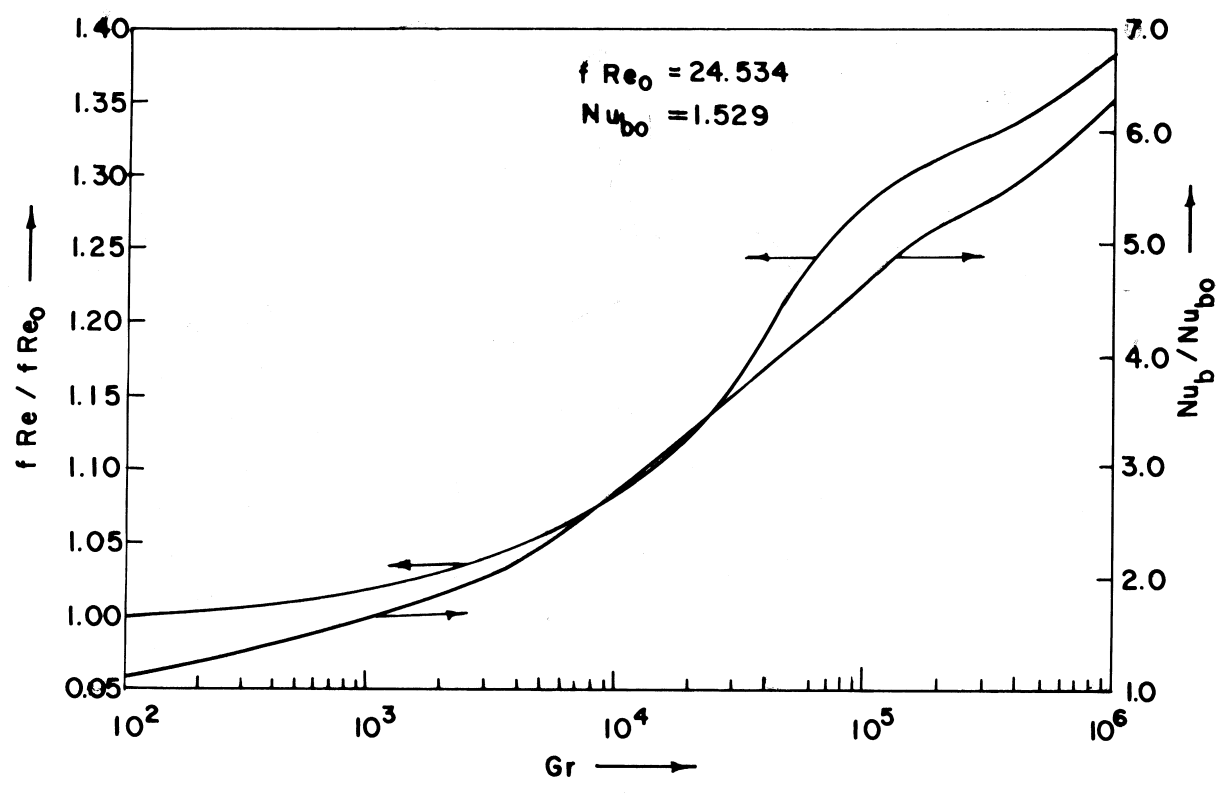

Fig. 7. Buoyancy effects on bundle average transport rates.

\subsection{Isotherms and streamlines}

The recirculating secondary flows in the $r, \theta$ plane generated due to buoyancy may be observed in Figs. 6(a) and (b). The isotherms are drawn on the left half and the streamlines on the right. Results for only two values of $G r=10^{3}$ and $10^{6}$ are presented here.

In the spaces between the symmetry lines and the adjacent rods, the buoyant flow moves along the heated surface 32 on the lower rod and along surface 14 on the upper rod to return along the cooler zones near the symmetry lines. The flow direction in both these zones is counterclockwise. On the other hand, clockwise streamlines are noted around the middle rod at $\theta=90^{\circ}$.

With increasing buoyancy, more number of loops are formed, Fig. 6(b) for $G r=10^{6}$. This implies that buoyancy causes increased mixing and, hence, higher $N u$ values, Fig. 2.

The isotherm values on the left half of the figures may be noted. The hottest zone occurs near the top of the central rod. The dimensionless temperature decreases as one proceeds to higher $\theta$ values resulting with $T^{*}<0$ near the bottom of the shell. A negative $T^{*}$ means that the local fluid temperature is less than the bundle average bulk temperature. This should be of no surprise, because the dimensionless bulk temperature has to be zero by definition, averaging between positive and negative values. Hence, a negative rod temperature does not restrict the rod to lose heat to the local fluid which is at a still lower temperature.

Note that the highest $T^{*}$ values decrease with increase in $G r$. This is so because the reference temperature $T_{\text {ref }}=q r_{\mathrm{i}} / k$ is also $G r$ dependent. However, the true temperature increases with $G r$. The isotherms intersect the shell orthogonally due to the adiabatic condition, and also the vertical symmetry lines because of angular symmetry.

\subsection{Transport rates}

The bundle average transport rates i.e. $f R e$ and $N u$ can be written as

$f R e=\frac{D_{\mathrm{h}}^{* 2}}{2 \bar{w}^{*}}$

and

$N u_{\mathrm{b}}=\frac{D_{\mathrm{h}}^{*}}{\bar{T}_{\mathrm{r}}^{*}-T_{\mathrm{b}}^{*}}$

The surface temperature averaged over all the seven rods, $\bar{T}_{\mathrm{r}}^{*}$ and the average axial velocity, $\bar{w}^{*}$ were determined numerically while the $T_{\mathrm{b}}^{*}$ is zero by definition.

The variations of transport rates over their pure forced flow values are plotted in Fig. 7. The observed trends are obvious from the previous discussions. With increasing $G r$, through-flow decreases and consequently $f R e$ increases. However, the increase is perceptible only beyond a $G r$ value of about $10^{3}$. In the experiments 
also, no measurable change was noticed in the $G r$ range of $1.7 \times 10^{2}$ to $2.2 \times 10^{3}$.

The bundle average $N u$, on the other hand, increases continuously with $\mathrm{Gr}$ as was presented in Fig. 2. Variations over a wider range are plotted in Fig. 7.

\section{Conclusions}

We modelled the circular geometry of the six peripheral rods by equivalent curved trapezoid. The forced convection transport rate values were found to be unchanged. The model afforded computational convenience through orthogonal intersections. The subsequent numerical solution helped in studying the flow and temperature fields. The highest temperature occurs at the top of the central rod. Increasing buoyancy decreases bulk flow rate and is reflected through increased friction at a given impressed axial pressure gradient. Buoyancy induced recirculating flow cells increase mixing, leading to higher heat transfer rate.

\section{References}

[1] M. Kaviany, Laminar combined convection in a horizon- tal annulus subject to constant heat flux inner wall and adiabatic outer wall, Trans. ASME, Paper No. 84-WA/ HT-49, 1884.

[2] A.O. Nieckele, S.V. Patankar, Laminar mixed convection in a concentric annulus with horizontal axis, Trans. ASME, J. Heat Transfer 107 (1985) 902-909.

[3] R.O. Warrington Jr, G. Crupper Jr, Natural convection heat transfer between cylindrical tube bundles and a cubical enclosure, in: Proc. Winter Annual Meeting, ASME, New York, 1979, pp. 143-148.

[4] C.J. Ho, W.S. Chang, C.C. Wang, Natural convection between two horizontal cylinders in an adiabatic circular enclosure, Trans. ASME, J. Heat Transfer 115 (1993) 158-165.

[5] R.W. Benodekar, A.W. Date, Numerical prediction of heat transfer characteristics of fully developed laminar flow through a circular channel containing rod clusters, Int. J. Heat Mass Transfer 21 (1978) 935-945.

[6] R. Das, A.K. Mohanty, Laminar combined convection in finite circular rod bundles, Trans. ASME, J. Heat Transfer 106 (1984) 563-569.

[7] A.K. Mohanty, S.C. Haldar, S. Sengupta, Low Reynolds number flow and heat transfer experiments in 7-rod vertical bundles, Nuclear Engineering and Design 143 (1993) 83-93. 